\title{
A Study Design to Evaluate Association between Smoking and Intracranial Atherosclerotic Stenosis
}

\author{
Hairi Liu, MD',5, Dong-geun Lee, MD', Seung Chul Jung, MD', Hyun Jung Koo, MD', \\ Eun Hye Kim, RN', Seon Moon Hwang, RT', Bum Joon Kim, MD², Yeonjung Kim, MD², \\ Hong Jun Cho, MD³, Min-ju Kim, Seung Hee Baek', Sang-Beom Jeon, MD², \\ Jong Sung Kim, MD², Dae Chul Suh, PhD'
}

Purpose: Smoking is a well known risk factor for stroke. The cerebral arteries may be uniquely susceptible to the atherosclerotic effects of smoking, such that it has a different risk profile for stroke compared with other atherosclerosis risk factors. It remains uncertain whether smoking is associated specifically with intracranial (IC) or extracranial (EC) atherosclerotic cerebrovascular disease. The aim of this study design will be to evaluate the association between smoking and severe IC stenosis, adjusting for other atherosclerosis risk factors, particularly age distribution.

Study design: This is a retrospective cohort study design. Participants are patients $(n=1714)$ with severe atherosclerotic stenosis undergoing cerebral catheter angiography because of stroke or transient ischaemic attack. All atherosclerotic steno-occlusive lesions are described in terms of location (anterior versus posterior circulation, IC versus EC, or intradural versus extradural). The atherosclerotic or stroke risk factors for analysis include age, gender, smoking history, number of lesions (single versus multiple), cardiac disease, diabetes mellitus, hypertension, family history, dyslipidemia, history of previous stroke, alcohol intake, metabolic syndrome and body mass index. Statistical analysis includes univariate analysis followed by multivariate logistic regression. The relationship between IC atherosclerotic stenosis and smoking will be assessed. Differences in risk factor distribution is analysed according to age at intervals of 10 years. Significant risk factors associated with IC atherosclerotic stenosis will also be assessed by multivariate logistic regression analysis.

Summary: This is an analytical study design that intends to measure the association between IC or EC atherosclerotic stenosis and smoking and other risk factors. We anticipate that it will have the power to detect any relationship between smoking and IC atherosclerotic lesions especially in younger patients.

Key Words : Smoking; Stenosis; Atherosclerosis; Risk Factors

'Departments of Radiology and Research Institute of Radiology, ${ }^{2}$ Department of Neurology, ${ }^{3}$ Department of Family Medicine, ${ }^{4}$ Department of Clinical Epidemiology and Biostatistics, University of Ulsan, College of Medicine, Asan Medical Center, Seoul, Korea; ${ }^{5}$ Department of Interventional Radiology, Taizhou People's Hospital, Taizhou, Jiangsu Province 225300, P. R. China

Received August 22, 2014; accepted after revision September 2, 2014.

Correspondence to: Dae Chul Suh, MD, Department of Radiology, University of Ulsan, College of Medicine, Asan Medical Center, 88, Olympic-ro 43-gil, Songpa-gu, Seoul 138-736, Korea.

Tel. 82.2.3010.4366 Fax. 82.2.476.0090 E-mail: dcsuh@amc.seoul.kr

This is an Open Access article distributed under the terms of the Creative Commons Attribution Non-Commercial License (http://creativecommons.org/licenses/by-nc/3.0) which permits unrestricted non-commercial use, distribution, and reproduction in any medium, provided the original work is properly cited. 


\section{Hairi Liu, et al.}

Intracranial (IC) atherosclerosis is more common in Asians than Caucasians. According to a study from South Korea, the distribution of symptomatic, severe stenotic lesions was $52 \%$ in IC arteries and $48 \%$ in extracranial (EC) arteries [1]. Single stenotic lesions were more likely to be located in an IC artery and were associated with a poor clinical outcome $[2,3]$. It is unclear why IC atherosclerosis is more common in Asians than Caucasians. Age, male gender and hyperlipidaemia were reported to favour EC atherosclerosis, while an association between metabolic syndrome and IC atherosclerosis was reported only for posterior circulation strokes [4]. IC stenotic lesions in young patients are predominantly located in the anterior circulation and occur more frequently in young women [5]. The presence of a lipid disorder was reported to be associated with the severity of the IC stenotic lesion, whereas the lesion's location depended on the nature of the risk factor and demographic features [6].

Cigarette smoke is an aerosol that contains $>4000$ chemicals, including nicotine, carbon monoxide, acrolein and oxidant compounds [7]. Smoking reduces flow-mediated dilatation (FMD) of systemic arteries in healthy young adults [8]. Smoking was reported to be a significant risk factor for carotid atherosclerosis and may accelerate the development and/or progression of atherosclerosis $[9,10]$. However, a study revealed that smoking and hypertension may be more closely associated with IC atherosclerosis than EC atherosclerosis after adjusting for potential confounding by other risk factors [11]. It should be noted that the criterion for significant cranial atherosclerotic stenosis in the previous studies was defined as being greater than 50\% as evaluated by magnetic resonance angiography, and the definition of the IC anatomical border was not described clearly.

Although smoking in Korea has decreased recently, the smoking rate in subjects aged over 20 years was about $26 \%$ (male versus female $=43.7 \%$ vs. $7.9 \%$ ) in 2012 [12]. Young adults who are involved in gambling are more likely to report cigarette smoking [13]. In South Korea, a large number of young adolescents spend approximately $2 \mathrm{~h}$ per day in computer game rooms, which may increase their exposure to tobacco smoke [14]. This is because smoking among Korean men is still a socially sanctioned behaviour in many indoor workplaces and commercial hospitality venues, including bars, nightclubs, restaurants and computer game rooms [14].

Few studies have examined the association between smoking and IC stenosis in young patients. IC stenosis was suggested to be more common in young patients, especially in those with a single, severe stenotic lesion $[11,15]$. In addition, the IC artery is not clearly defined anatomically. Therefore, we are planning to elucidate the association between smoking and IC atherosclerosis based on an exact anatomical location defined angiographically. Furthermore, we aim to examine whether this association is confounded by age, number of lesions and other atherosclerotic risk factors.

\section{MATERIALS AND METHODS}

\section{Overall design}

Participants in this retrospective cohort study include patients with severe cerebral artery atherosclerotic stenosis or occlusion undergoing cerebral angiography because of stroke or transient ischaemic attack. The diagnosis of occlusion or the degree of stenosis is made using high-resolution, biplane digital subtraction angiography (DSA) (Siemens Axiom Artis Zee biplane angiography system, Siemens AG, Medical Solutions, Erlangen, Germany) of the internal carotid artery (ICA), common carotid artery (CCA), vertebral artery (VA) and/or proximal subclavian artery. All lesions are describe in terms of location (anterior (AC) vs. posterior (PC) circulation, IC vs. EC and intradural (ID) vs. extradural (ED)).[16] Risk factors for atherosclerosis or stroke include age, gender, smoking history, number of lesions (single or multiple), cardiac disease, diabetes mellitus (DM), hypertension (HTN), family history of stroke, hyperlipidaemia, previous stroke, alcohol intake, metabolic syndrome and body mass index (BMI). The independent association between all risk factors and the outcome of IC atherosclerotic stenosis is assessed using univariable analysis. IC atherosclerotic stenosis is then modelled using multiple logistic regression, with smoking as the explanatory variable, examining for potential confounding by all risk factors that survived after univariable analysis.

The Institutional Review Board approves the study design and the use of clinical data, and all patients provide written informed consent for the angiographic procedures. Because of the retrospective and observational nature of this study, the need for written informed consent for retrospective analysis is waived.

\section{Patients}

We review the records of 1714 consecutive patients diagnosed with atherosclerotic severe stenosis or 
occlusion involving a cerebral artery in prospective neurointervention database at Asan Medical Centre, Seoul, Korea between January 2002 and December 2012.

Patients aged between 30 and 80 years old are included in the study. Among patients who underwent cerebral angiography due to TIA/stroke, severe stenosis of cerebral artery $\geq 70 \%$ or occlusion in the carotid artery, the VA and the subclavian artery and the IC cerebral arteries to the A1, M1 and P1-2 lesions are included [17-19]. We assume that severe stenosis of the cerebral artery $\geq 70 \%$ or occlusion is clinically the most critical degree of stenosis related with certain and definite pathophysiology related with presenting symptom and prognosis. Mild to moderate stenosis is excluded because mild to moderate stenosis is a common finding on cerebral catheter angiography in the elderly patients, even in asymptomatic patients and clinical significant is uncertain.

The following patient groups are excluded: patients aged $>80$ or $<30[20]$; patients with lesions located beyond A1 of ACA, M1 of MCA or P2 of PCA; patients who had undergone revascularisation with thrombolysis or thrombectomy because of acute onset of symptoms, dissection or other vascular disease such as vasculitis or moyamoya disease, restenosis after stenting/angioplasty, or endarterectomy of extra endovascular removal of a cardiac embolism. The presence of cardioembolism is determined by angiographic finding of embolism as a filling defect in the vessel and also underlying severe atherosclerotic stenosis or occlusion as well as cardiac evaluation for the source of the embolism [17, 19, 21, 22].

\section{DEFINITIONS}

\section{Patient age groups}

Patients are classified as being young (30?55 years) or old ( $>55-80$ years) at the time of DSA examination [23]. We also determine the age distribution in 10 year intervals.

\section{Stenosis location in the ICA}

To aid the precise identification of a lesion's location, the ICA is divided into its embryological vascular segment and corresponding remnant branch. ICA segments are then defined based on the three anatomical parts of occlusion levels: supraclinoid-terminal (Supra-T), petrocavernous (PC) and bulb-cervical (BC) [16].

\section{Single vs. multiple lesions}

All patients underwent selective angiography of the ICA, CCA, VA and/or proximal subclavian artery. A single lesion is defined as when there is only one occurrence of a severe cerebral artery stenotic lesion ( $\geq 70 \%$ ) or occlusion on cerebral angiography. The presence of multiple lesions is defined as the occurrence of at least two severe stenotic lesions or occlusions. In patients with multiple lesions, the one responsible for the patient's symptom presentation is deemed to be their main lesion.

\section{Angiographic evaluation of lesion location (IC vs. EC and ID vs. ED)}

The junction between ID and ED arteries is set as the angiographic dural margin just below the ophthalmic artery. The junction between IC and EC arteries is set as the ICA segment of the lower margin of the carotid canal in the petrous bone for the ICA, and as the level of foramen magnum for the VA [16].

\section{Smoking}

Smoking definition and classification follows those of the US Centers for Disease Control and Prevention. Non-smokers are defined as those who currently do not smoke cigarettes, including both former smokers and never smokers. Never smokers are defined as those who have never smoked or who have smoked fewer than 100 cigarettes in their entire lifetime. Former smokers are defined as those who have smoked at least 100 cigarettes in their lifetime but currently do not smoke. Current smokers are defined as those who have smoked 100 cigarettes in their lifetime and currently smoke cigarettes every day (daily) or some days (nondaily) [24]. Heavy smokers are defined as those who smoke 20 or more cigarettes per day, or 20 or more pack-years according to a recent report in the International Journal of Environmental Research and Public Health [25].

\section{Other risk factors}

Cardiac disease risk factors include myocardial infarction, angina and a history of coronary artery bypass graft or percutaneous coronary intervention. Diagnosis of DM is established if the patient met at least one of the following criteria [26]: (1) past history of known DM; (2) glycated haemoglobin (HbA1c) $\geq$ $6.5 \%$; (3) serum glucose level after an $8 \mathrm{~h}$ fast $\geq 126$ $\mathrm{mg} / \mathrm{dL}$; (4) glucose level $2 \mathrm{~h}$ after a 75 goral glucose tolerance test $\geq 200 \mathrm{mg} / \mathrm{dL}$; and (5) serum glucose level $\geq 200 \mathrm{mg} / \mathrm{dL}$ on random testing. Diagnosis 


\section{Hairi Liu, et al.}

of hypertension is established if the patient had a history of known hypertension (systolic blood pressure $\geq 140 \mathrm{mmHg}$ and/or diastolic blood pressure $\geq 90 \mathrm{~mm}$ $\mathrm{Hg}$ ) or is on antihypertensive treatment. Diagnosis of dyslipidaemia is established if the patient met at least one of the following criteria: (1) past history of known hyperlipidaemia and on hyperlipidaemia treatment; (2) total cholesterol $\geq 200 \mathrm{mg} / \mathrm{dL}$; (3) triglycerides $\geq 200$ $\mathrm{mg} / \mathrm{dL}$; and (4) low-density lipoprotein $\geq 130 \mathrm{mg} / \mathrm{dL}$ (5) high-density lipoprotein $\leq 40 \mathrm{mg} / \mathrm{dL}$.

Stroke includes having a past history of ischaemic stroke, haemorrhagic stroke or transient ischaemic attack. Family history is recorded as positive if apparent or sibling suffered IC or EC atherosclerotic stenosis. A history of alcohol intake is defined as those who currently drank alcohol or who had quit drinking less than 6 months previously. BMI $\geq 30 \mathrm{~kg} / \mathrm{m}^{2}$ is defined as a positive risk factor.

Metabolic syndrome is defined by the criteria of the National Cholesterol Education Program as the presence of three or more of the following: (1) abdominal obesity; (2) elevated triglyceride levels ( $\geq 150$ $\mathrm{mg} / \mathrm{dL}$ ); (3) decreased high-density lipoprotein cholesterol (HDL-C) levels ( $<40 \mathrm{mg} / \mathrm{dL}$ for men and $<50$ $\mathrm{mg} / \mathrm{dL}$ for women); (4) high blood pressure (systolic blood pressure $\geq 130 \mathrm{mmHg}$, diastolic blood pressure $\geq 85 \mathrm{mmHg}$ or use of antihypertensive medication); and (5) elevated fasting glucose levels ( $\geq 110$ $\mathrm{mg} / \mathrm{dL}$ ).[27] Abdominal obesity is defined as a waist circumference of $\geq 90 \mathrm{~cm}$ for men and $\geq 80 \mathrm{~cm}$ for women according to the revised Asia-Pacific criteria suggested by the World Health Organization Western Pacific Region [28].

\section{Clinical data collection}

The physician responsible for consideration of the patient for DSA fills out a standardised data collection form to collect information on age, gender, pattern of cerebral atherosclerosis (lesion location and severity), cardiac disease, DM, HTN, family history, dyslipidaemia, previous stroke history, alcohol history, metabolic syndrome and BMI, medical history and current medication. Patients' angiographic findings and medical records are recorded prospectively to assess patient demographics; however, additional smoking history is assessed retrospectively by telephone to obtain the data in sufficient detail.

\section{Statistics and Study outcome}

Distribution of patient age, gender, lesion location (AC vs. PC, IC vs. EC, and ID vs. ED) and number (single vs. multiple) are correlated with the history of smoking. We also evaluate the proportion of patients with IC atherosclerotic stenosis among smokers and compare this with the proportion in patients stratified by the other risk factors. Differences in risk factor distribution are analysed according to age in 10 year intervals.

Categorical variables are presented as frequencies and percentages, and continuous variables are expressed as mean and SD. The $t$ test is used to compare continuous variables, and the chi-square test or Fisher exact test are used to compare categorical variables, as appropriate. Associations between risk factors (age, gender, location, multiplicity, smoking, etc.) and IC atherosclerosis stenosis are tested by using the univariate logistic regression model followed by the multivariable logistic regression model with backward elimination. All reported $\mathrm{p}$-values are two sided, and $\mathrm{P}<.05$ is considered statistically significant. Statistical analyses are conducted using SPSS 21 software (SPSS Inc., Chicago, IL, USA).

\section{SUMMARY}

This analytical cohort study is designed to investigate the association between IC atherosclerotic stenosis and smoking, adjusting for confounding by other risk factors. We anticipate that it will have the power to detect any relationship between smoking and IC atherosclerotic lesions especially in younger patients.

\section{References}

1. Suh DC, Lee SH, Kim KR, Park ST, Lim SM, Kim SJ, et al. Pattern of atherosclerotic carotid stenosis in Korean patients with stroke: different involvement of intracranial versus extracranial vessels. AJNR Am J Neuroradiol 2003;24:239-244

2. Pyun HW, Suh DC, Kim JK, Kim JS, Choi YJ, Kim MH, et al. Concomitant multiple revascularizations in supra-aortic arteries: short-term results in 50 patients. AJNR Am J Neuroradiol 2007;28:1895-1901

3. Liu S, Jung JH, Kim SM, Lim HK, Kwon HJ, Kim JK, et al. Simultaneous bilateral carotid stenting in high-risk patients. AJNR Am J Neuroradiol 2010;31:1113-1117

4. Kim JS, Nah HW, Park SM, Kim SK, Cho KH, Lee J, et al. Risk factors and stroke mechanisms in atherosclerotic stroke: intracranial compared with extracranial and anterior compared with posterior circulation disease. Stroke 2012;43:3313-3318

5. Siddiq F, Chaudhry SA, Vazquez G, Suri MF, Qureshi AI. Intracranial stenosis in young patients: unique characteristics and risk factors. Neuroepidemiology 2012;38:148-153

6. Turan TN, Makki AA, Tsappidi S, Cotsonis G, Lynn MJ, Cloft HJ, et al. Risk factors associated with severity and location of intracranial arterial stenosis. Stroke 2010;41:1636-1640

7. Csordas A, Bernhard D. The biology behind the atherothrombotic 


\section{Association of Smoking with Intracranial Stenosis}

effects of cigarette smoke. Nat Rev Cardiol 2013;10:219-230

8. Celermajer DS, Sorensen KE, Gooch VM, Spiegelhalter DJ, Miller OI, Sullivan ID, et al. Non-invasive detection of endothelial dysfunction in children and adults at risk of atherosclerosis. Lancet 1992;340:1111-1115

9. Redgrave JN, Lovett JK, Rothwell PM. Histological features of symptomatic carotid plaques in relation to age and smoking: the oxford plaque study. Stroke 2010;41:2288-2294

10. Kweon SS, Lee YH, Shin MH, Choi JS, Rhee JA, Choi SW, et al. Effects of cumulative smoking exposure and duration of smoking cessation on carotid artery structure. Circ J 2012;76:2041-2047

11. Kim DE, Lee KB, Jang IM, Roh H, Ahn MY, Lee J. Associations of cigarette smoking with intracranial atherosclerosis in the patients with acute ischemic stroke. Clin Neurol Neurosurg 2012;114:1243-1247

12. Choi SH. Trends of smoking status in South Korea. Public Health Weekly Report;7(22):478-480

13. Hayatbakhsh MR, Clavarino A, Williams GM, Bor W, Najman JM. Young adults' gambling and its association with mental health and substance use problems. Aust N Z J Public Health 2012;36:160-166

14. Kim S, Sohn J, Lee K. Exposure to particulate matters (PM2.5) and airborne nicotine in computer game rooms after implementation of smoke-free legislation in South Korea. Nicotine Tob Res 2010;12:1246-1253

15. Song YM, Cho HJ. Risk of stroke and myocardial infarction after reduction or cessation of cigarette smoking: a cohort study in korean men. Stroke 2008;39:2432-2438

16. Kwak JH, Zhao L, Kim JK, Park S, Lee DG, Shim JH, et al. The outcome and efficacy of recanalization in patients with acute internal carotid artery occlusion. AJNR Am J Neuroradiol 2014;35:747-753

17. Lu PH, Park JW, Park S, Kim JL, Lee DH, Kwon SU, et al. Intracranial stenting of subacute symptomatic atherosclerotic occlusion versus stenosis. Stroke 2011;42:3470-3476

18. Suh DC, Kim JK, Choi JW, Choi BS, Pyun HW, Choi YJ, et al. Intracranial stenting of severe symptomatic intracranial stenosis: results of 100 consecutive patients. AJNR Am J Neuroradiol
2008;29:781-785

19. Suh DC, Kim JK, Choi CG, Kim SJ, Pyun HW, Ahn C, et al. Prognostic factors for neurologic outcome after endovascular revascularization of acute symptomatic occlusion of the internal carotid artery. AJNR Am J Neuroradiol 2007;28:1167-1171

20. Chimowitz MI, Lynn MJ, Turan TN, Fiorella D, Lane BF, Janis S, et al. Design of the stenting and aggressive medical management for preventing recurrent stroke in intracranial stenosis trial. $J$ Stroke Cerebrovasc Dis 2011;20:357-368

21. In HS, Lee HY, Park JY, Kim SY, Jung JH, Kim JS, et al. Intracranial stenting in patients with atherosclerotic stenosis associated with various aneurysms in the same diseased arterial segment. AJNR Am J Neuroradiol 2010;31:1895-1898

22. Choi JW, Kim JK, Choi BS, Kim JH, Hwang HJ, Kim JS, et al. Adjuvant revascularization of intracranial artery occlusion with angioplasty and/or stenting. Neuroradiology 2009;51:33-43

23. Thun MJ, Carter BD, Feskanich D, Freedman ND, Prentice R, Lopez AD, et al. 50-year trends in smoking-related mortality in the United States. N Engl J Med 2013;368:351-364

24. Blackwell DL, Lucas JW, Clarke TC. Summary health statistics for U.S. Adults: national health interview survey, 2012. Vital Health Stat 10 2014:1-171

25. Tchounwou PB, Han O. International Journal of Environmental Research and Public Health Best Paper Award 2013. Int J Environ Res Public Health 2013;10:443-445

26. Lee DG, Zhao LB, Shim JH, Lee DH, Suh DC. Relationship between diabetes mellitus with dural arteriovenous fistula. Neuroradiology 2013;55:1129-1134

27. Expert Panel on Detection E, Treatment of High Blood Cholesterol in A. Executive Summary of The Third Report of The National Cholesterol Education Program (NCEP) Expert Panel on Detection, Evaluation, And Treatment of High Blood Cholesterol In Adults (Adult Treatment Panel III). JAMA 2001;285:2486-2497

28. Kanazawa M, Yoshiike N, Osaka T, Numba Y, Zimmet P, Inoue S. Criteria and classification of obesity in Japan and Asia-Oceania. World Rev Nutr Diet 2005;94:1-12 